\title{
An evidence map of the effect of Tai Chi on health outcomes
}

\author{
Michele R. Solloway', Stephanie L. Taylor ${ }^{1}$, Paul G. Shekelle ${ }^{1,2,3,4}$, Isomi M. Miake-Lye ${ }^{1,2}$, Jessica M. Beroes², \\ Roberta M. Shanman ${ }^{4}$ and Susanne Hempel ${ }^{4^{*}}$ (D)
}

\begin{abstract}
Background: This evidence map describes the volume and focus of Tai Chi research reporting health outcomes. Originally developed as a martial art, Tai Chi is typically taught as a series of slow, low-impact movements that integrate the breath, mind, and physical activity to achieve greater awareness and a sense of well-being.

Methods: The evidence map is based on a systematic review of systematic reviews. We searched 11 electronic databases from inception to February 2014, screened reviews of reviews, and consulted with topic experts. We used a bubble plot to graphically display clinical topics, literature size, number of reviews, and a broad estimate of effectiveness.

Results: The map is based on 107 systematic reviews. Two thirds of the reviews were published in the last five years. The topics with the largest number of published randomized controlled trials (RCTs) were general health benefits (51 RCTs), psychological well-being (37 RCTs), interventions for older adults (31 RCTs), balance (27 RCTs), hypertension (18 RCTs), fall prevention (15 RCTs), and cognitive performance (11 RCTs). The map identified a number of areas with evidence of a potentially positive treatment effect on patient outcomes, including Tai Chi for hypertension, fall prevention outside of institutions, cognitive performance, osteoarthritis, depression, chronic obstructive pulmonary disease, pain, balance confidence, and muscle strength. However, identified reviews cautioned that firm conclusions cannot be drawn due to methodological limitations in the original studies and/or an insufficient number of existing research studies.
\end{abstract}

Conclusions: Tai Chi has been applied in diverse clinical areas, and for a number of these, systematic reviews have indicated promising results. The evidence map provides a visual overview of Tai Chi research volume and content.

Systematic review registration: PROSPERO CRD42014009907

Keywords: Systematic review, Tai Chi, Evidence map, Health

\section{Background}

Tai Chi, also known as T'ai chi ch'uan or Taijiquan, developed as an ancient Chinese martial art and is today widely practiced for its health benefits. Many forms of Tai Chi exist, but in western culture, it is most commonly taught as a series of slow, gentle, low-impact movements that integrate the breath, mind, and physical activity to achieve greater awareness and a sense of inner peace and well-being. The meditative movement is designed to strengthen and stretch the body, improve the flow of blood and other fluids, improve balance, proprioception, and awareness of how the body moves

\footnotetext{
* Correspondence: susanne_hempel@rand.org

${ }^{4}$ Evidence-based Practice Center (EPC), RAND Corporation, Santa Monica, CA, USA Full list of author information is available at the end of the article
}

through space; and it may be practiced in a group format or alone [1]. Results from the 2007 National Health Interview Survey-a survey of a representative sample of adults in the USA-estimated that approximately 2.3 million adults in the USA practiced Tai Chi in the past 12 months. There is no official licensure granted by national or state professional boards, and there are no official standards for training instructors; thus, individual training programs vary.

Research on effects of Tai Chi on health outcomes continues to expand and has been the subject of many primary research studies and reviews of the literature. The research field covers a wide spectrum of clinical indications, targets a range of populations, and has focused on a variety of settings. A systematic review of systematic 
reviews identified 35 reviews published in 2010 and concluded that Tai Chi is effective for fall prevention and improving psychological health and was associated with general health benefits for older people [2]. However, the interest in Tai Chi has increased in particular in recent years and since 2010, more than twice as many systematic reviews have been published. In order to provide a broad overview of the research evidence that has been published to date, we conducted a systematic review of systematic reviews of the effects of Tai Chi on health outcomes [3].

We present the results of the systematic review of systematic reviews as an evidence map, a form of systematic literature synthesis that uses visual displays of the volume and content areas of research. Evidence maps are an emerging evidence synthesis tool that aim to provide an overview over large research areas [4]. The evidence map presents a summary of the focus of Tai Chi research that contributes to the evidence base on patient health outcomes in a format that is easily accessible to healthcare practitioners and policy makers and other stakeholders. The objective of the evidence map is to indicate the research focus and show the presence as well as the absence of published research for individual topic areas; the evidence map may inform research agendas or be used as a signpost for practitioners.

\section{Methods}

The evidence map is based on a systematic review of systematic reviews and summarizes healthcare research reporting on patient health outcomes on effects of Tai Chi. Systematic reviews provide comprehensive summaries of the literature for defined clinical topics by combining thorough and comprehensive searches and transparent synthesis of the available evidence. Systematic reviews often employ meta-analysis which provides the statistical power to identify small treatment effects by combining often small and underpowered studies.

We have registered this systematic review in PROSPERO (record number CRD42014009907). We report one deviation from the protocol: systematic reviews that do not include randomized controlled trials (RCTs) were not summarized in a narrative synthesis but were included in the bubble plot in the unclear category of the $x$-axis with the $y$-axis indicating that no RCT was identified despite an explicit search (see the "Data synthesis" section for more information). This manuscript is based on a comprehensive report for the Department of Veterans Affairs (VA), Veterans Health Administration, Office of Research and Development, Quality Enhancement Research Initiative, conducted within the Evidence-based Synthesis Program of the VA [3]. This manuscript aims to disseminate the finding to a broader audience of interested stakeholders. We report the methodology and results of the evidence map according to the PRISMA guidelines for systematic reviews to the extent possible (the PRISMA checklist is documented in Additional file 1). The VA report discusses the results within the context of the VA healthcare system and includes additional evidence synthesis results for VA-identified priority areas. This evidence map was supported by a technical expert panel of practitioner, policy maker, and researcher content experts.

\section{Data sources}

We searched PubMed, CINAHL, Database of Abstracts of Reviews of Effects (DARE), Cochrane Database of Systematic Reviews (CDSR), Health Technology Assessments (HTA), Economic Evaluations (EED), Allied and Complementary Medicine (AMED), PsycINFO, Scopus, Web of Science, and PROSPERO from database inception to February 2014 for published English-language systematic reviews. In addition, we screened published reviews of reviews and consulted with topic experts. We used the terms "tai chi," "tai-chi," "tai ji," "tai-ji," "taiji," "t'ai chi," "t' ai chi," "taijiquan," and "shadow boxing;" the full search strategy is documented in Additional file 2.

\section{Inclusion criteria \\ Design}

Systematic reviews focusing on Tai Chi and summarizing primary research studies for all clinical indications were eligible for inclusion. We defined systematic reviews as reviews that either self-identified as a "systematic review" or reviews that reported the search sources and accounted for identified studies.

\section{Participants}

Systematic reviews of adult participants or unspecified age groups regardless of their health status were eligible for inclusion in the review; systematic reviews exclusively focusing on children and adolescents were excluded.

\section{Intervention}

Systematic reviews of the effects of Tai Chi for any clinical indication were eligible for inclusion. Systematic reviews addressing Tai Chi and other approaches were eligible if one of the two following criteria was met: (a) "Tai Chi" was part of the search strategy or (b) the search strategy did not specify any interventions (e.g., focused on an outcome) and the systematic review identified Tai Chi studies. We excluded systematic reviews that included Tai Chi studies but did not systematically search for these (e.g., by reviewing "exercise" interventions where only those Tai Chi studies were found that used the descriptive term "exercise") and broad reviews on complementary and alternative medicine approaches without particular focus on Tai Chi. 


\section{Outcome}

Systematic reviews reporting on patient health outcomes were eligible for inclusion. Systematic reviews of provider outcomes, acceptance, prevalence, use, costs, study design features, or intervention features not reporting patient health outcomes were excluded.

\section{Timing}

Systematic reviews summarizing evaluations of interventions of any duration and follow-up point were eligible for inclusion.

\section{Setting}

Systematic reviews of studies in healthcare-related settings were eligible for inclusion. English-language systematic reviews, regardless of the language of the included studies were eligible for inclusion.

\section{Procedure}

Two independent literature reviewers screened the systematic review search output. Citations deemed potentially relevant by at least one reviewer and unclear citations were obtained as full text. The full-text publications were screened against the specified inclusion criteria by two independent reviewers; disagreements were resolved through discussion. The reasons for exclusion of full-text publications were recorded (Fig. 1). Where originals and updates of systematic reviews by the same author group were available, only the most recent version was considered, and multiple publications of the same review were counted as one review but data were extracted from all available publications [5-8]. From each included systematic review, we extracted the specific clinical indication (e.g., osteoarthritis) and the main patient outcomes (e.g., balance) that were summarized across included studies. We extracted the number of Tai

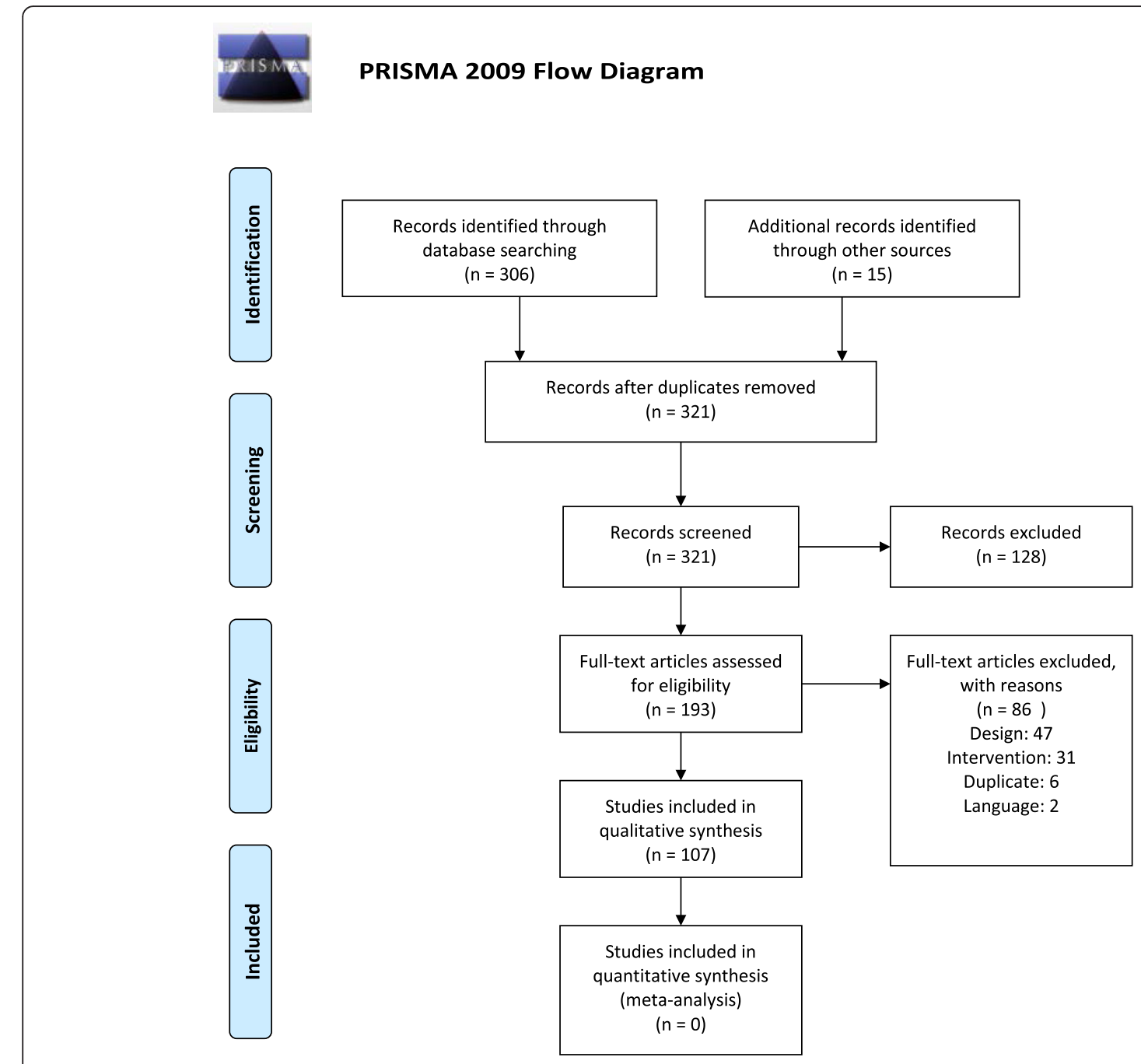

Fig. 1 Literature flow 
Chi RCTs included in the review, outcomes measured, comparators, treatment effect estimates for patient outcomes, and review characteristics. In addition, we documented which reviews were based on a format of Tai Chi that deviated from traditional formats (e.g., no weight shifting component; water-based; sitting, not standing; limited training intensity).

\section{Data synthesis}

We used a bubble plot to visually display the Tai Chi research field.

\section{Clinical indications (bubbles)}

We used the topics of the identified systematic reviews to categorize the reviews. Reviews focused on outcomes, populations, or clinical indications. Systematic reviews groupings into clinical topics were drafted by one reviewer and discussed in the review team. Decisions not to combine potentially related topic areas (e.g., reviews on the outcome hypertension and reviews on patients with cardiovascular disease) were based on the lack of overlap between studies included in the reviews, differences in the reported outcomes, and differences in the review's conclusion. All identified systematic reviews were allocated to a single content area and were only depicted once on the bubble plot.

\section{Color}

Indications that have been addressed in a publication by an agency specializing in unbiased evidence syntheses such as Cochrane and the Agency for Healthcare Research and Quality (AHRQ) are shown in dark green (all other bubbles are pale yellow).

\section{Number of reviews (bubble size)}

We used the size of the bubble to represent the number of systematic reviews on the topic

\section{Literature size estimate (y-axis)}

The bubble plot provides an overview of the research volume for each of the identified clinical indications. We used the number of RCTs per review, selecting the systematic review with the most included Tai Chi RCTs for the individual topic as the research volume estimate.

\section{Effect estimate ( $x$-axis)}

The bubble plots provide a very broad indication of the clinical effectiveness of Tai Chi according to patient health outcomes reported in RCTs. All available systematic reviews were reviewed for each clinical indication noted on the evidence map. Greater significance was attributed to the largest review as it should provide the most complete literature synthesis, and reviews from agencies specializing in unbiased evidence syntheses as it should provide the most valid synthesis. Reviews reporting on only one RCT were classified as unclear evidence regardless of the statistical significance of the individual study given the paucity of the existing research and lack of replication of effect. For effect size estimates, metaanalytic results were sought to provide a summary effect across individual and often small and underpowered studies. Reviews reporting on studies with conflicting results across studies were classified as unclear evidence unless they reported a statistically significantly positive pooled effect estimate favoring Tai Chi or all included studies reported a positive effect of Tai Chi. The evidence map is divided into three sections: topics with evidence indicating potentially no effect (left section); topics for which the evidence base is unclear (middle section); and topics for which there is published evidence of a potential positive effect with a meta-analysis reporting statistically significant treatment effects of Tai Chi (right section).

\section{Results}

We identified 321 citations of which 107 unique systematic reviews met the criteria for inclusion in the review [5, 7, 9-113]. Figure 2 provides a graphic representation of the evidence base. Two thirds (66\%) of the reviews were published in the last five years and spanned a wide diversity of clinical indications, study populations, and outcomes.

\section{Size of research base}

Topics with the largest research base included research on general health effects, psychological well-being, interventions in older adults, and effects on the outcome balance, hypertension, falls prevention, and cognitive performance.

The evidence base for the effectiveness of Tai Chi was unclear for the five of the largest areas of research. Six systematic reviews addressed positive effects of Tai Chi on health outcomes (Health) [7, 16, 36, 55, 59, 78]. The largest review, a comprehensive review of health benefits of qigong and Tai Chi, included 51 RCTs but did not provide treatment effect estimates across individual studies [55] and the other reviews primarily highlighted the need for more research. The reviews included studies that addressed a large range of outcomes. The largest review included studies that reported on 163 physiological and psychological health outcomes [55]. An AHRQ evidence report on meditation practices required studies to report measurable data for health-related outcomes and differentiated physiological (e.g., sensory outcomes), psychosocial (e.g., social and interpersonal relationships), and clinical outcomes (e.g., longevity) [7]. The other reviews differentiated the outcome categories balance improvement/postural stability/fall prevention, cardiovascular and ventilator enhancement, and other outcomes (rheumatoid arthritis, pain reduction, stress 


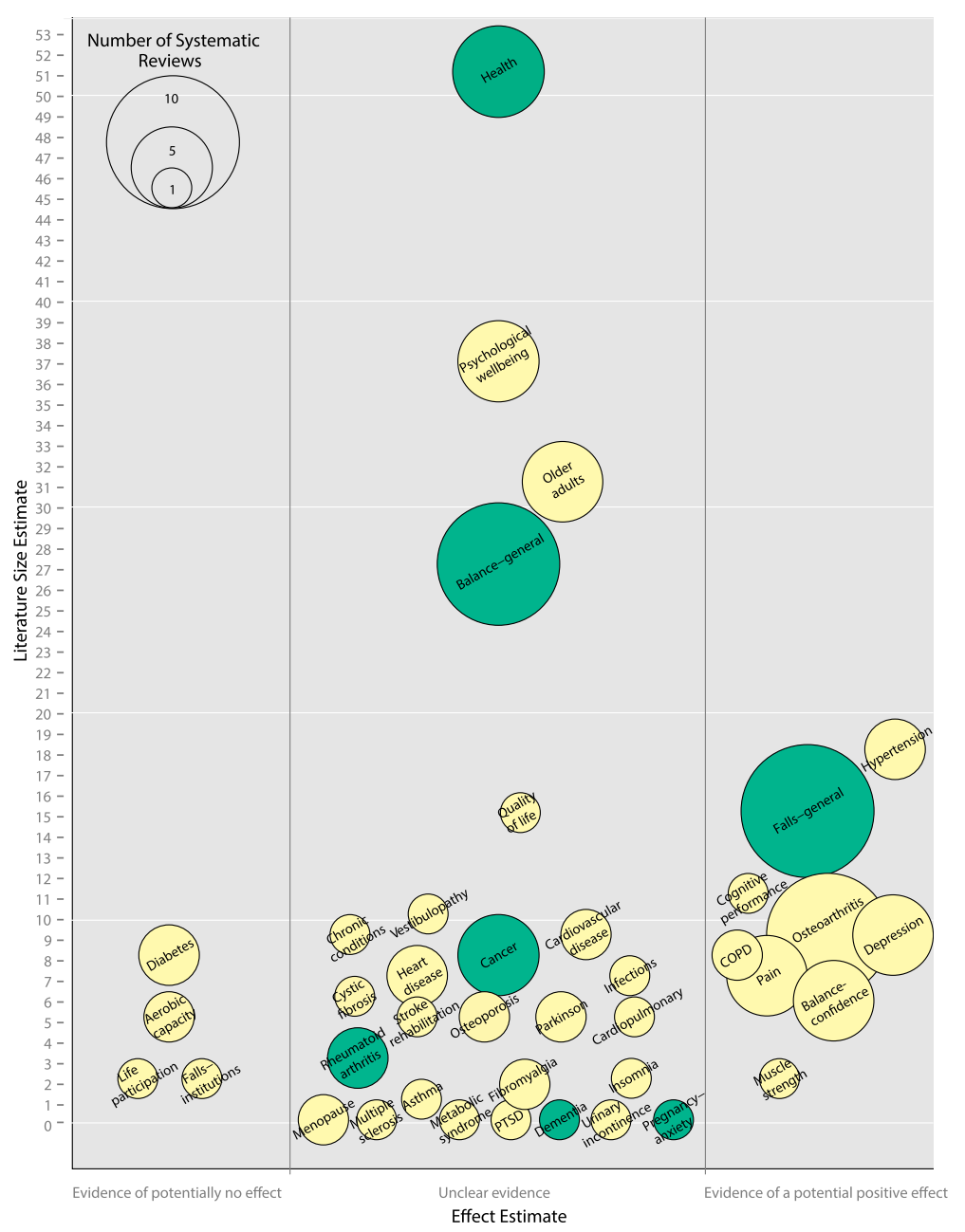

Fig. 2 Evidence map of Tai Chi. The bubble plot displays Tai Chi research based on systematic reviews published to February 2014. $y$-axis: literature size estimate (number of RCTs included in the largest systematic review). $x$-axis: effect estimate (three partitions: evidence of potentially no effect, unclear evidence base, evidence of a potential positive effect). Bubbles: clinical indication. Color: green bubbles indicate that the identified systematic reviews on the topic include a Cochrane review or an AHRQ evidence report. Bubble size: number of systematic reviews on the topic

reduction, nightmare reduction); [16] included studies reporting on health outcomes such as cardio respiratory function, falls, balance, strength, or quality of life; [36] reported more than 22 different outcomes addressed in included studies and highlighted effects on quality of life, physical functioning, pain management, balance and risk of falls reduction, enhancing immune response, and improving flexibility/strength/kinesthetic sense; [59] or differentiated effects on cardiovascular disease, chronic disease and immunity, and psychological benefits [78].

Five systematic reviews concentrated on psychological well-being [24, 32, 56, 95, 111]. The largest review included 37 RCTs, but treatment estimates were only presented for three of the included RCTs [24]. A metaanalysis reported positive pooled results for a few selected outcomes, [95] two reviews did not provide specific treatment estimates, $[56,111]$ and one concluded that it is premature to form conclusions on the effect of Tai Chi on psychological well-being [32]. Four published systematic reviews have examined Tai Chi and qigong for older adults $[84,85,94,113]$. The review authors focused specifically on this population and did not restrict the reviews to a particular clinical outcome. The reviews addressed a range of outcomes and analyses, including perceived benefits to health, perceived improved mediators such as social support, and perceived factors for initiating Tai Chi; [84] the efficacy of Tai Chi Chuan based on outcomes reported in included studies such as falls, balance, or cardiorespiratory functions; [94] physical and psychological health outcomes differentiating identified outcomes into the categories falls and balance, physical function, cardiovascular disease, and psychological and additional disease-specific responses; [85] and validated measures and self-reported indicators of mental well-being such as life satisfaction, 
mental health-related quality of life, self-esteem, or happiness and mastery [113]. The largest review included 31 RCTs; [85] none of the reviews reported specific treatment estimates for Tai Chi across studies.

The outcome balance has been addressed in nine systematic reviews by independent author groups $[10,37$, $39,53,57,61,72,77,109]$ and the largest review included 27 RCTs. The largest review did not report treatment effect estimates [57] and an existing Cochrane review on exercise interventions included 12 Tai Chi RCTs but reported effects only for a combination of Tai Chi, gi gong, dance, and yoga interventions [53]. Another review pooled three RCTs and found no effect of Tai Chi on the single leg stance test compared to different control groups [10] while results of studies included in the remaining reviews varied and none of the reviews provided a treatment effect estimate across identified studies. A systematic review addressing health-related quality of life included 15 Tai Chi RCTs [28] but did not provide a summary estimate for Tai Chi effects and individual study results varied within and across studies.

\section{Potentially promising effects}

Promising effects of Tai Chi, indicated by statistically significant pooled treatment effects in systematic reviews, and based on a substantial number of research studies included findings for hypertension, falls prevention outside of institutions, and cognitive performance. Hypertension has been addressed in three systematic reviews [68, 97, 106]. The pooled results of the largest review (18 RCTs) showed a larger number of participants with reduced blood pressure (relative risk [RR] 3.39; $95 \%$ confidence interval $[\mathrm{CI}] 1.81,6.34 ; 4 \mathrm{RCTs})$; reduced mean systolic blood pressure (mmHg WMD 12.43; 95 \% CI 12.24, 12.62; 10 RCTs); and reduced mean diastolic blood pressure (mmHg WMD 6.03; 95 \% CI 5.90, 6.16; 10 RCTs) compared to usual care [97]. However, the authors cautioned that the evidence remains weak and stated reservations due to the poor quality of the included studies, lack of longer follow-up, or conflicting results across outcomes, comparators, and settings. An earlier review that included only four RCTs in elderly participants concluded that the evidence for Tai Chi in reducing blood pressure in the elderly is limited, [68] and the third review did not provide a pooled treatment estimates across studies [106]. Tai Chi for fall prevention in unselected populations or participants living in the community (Falls-general) has been addressed in ten independent reviews [17, 20, 43, 47, 48, $51,73,75,87,101]$. The largest review (15 RCTs) reported no benefit compared to non-exercise controls across five studies but found a significant pooled estimate for Tai Chi versus exercise controls (incidence rate ratio [IRR] 0.51; $95 \%$ CI $0.38,0.68 ; 2$ RCTs); the review discussed a number of explanations for this finding, including a dose-response effect [73]. A Cochrane review on interventions for preventing falls in older people living in the community found no reduction in the rate of falls but reported a significantly reduced risk of falling (RR $0.71 ; 95 \%$ CI 0.57, 0.87; 6 RCTs) associated with Tai Chi compared to diverse, predominantly passive comparators (e.g., wellness education) [47]. An AHRQ report on interventions to prevent falls in older adults included three Tai Chi RCTs, but no summary treatment effect was reported [20]. A further review reported a statistically significant pooled estimate for Tai Chi in communitydwelling participants (RR 0.66; $95 \%$ CI 0.52, 0.78; comparators not specified) [17]. One review found no Tai Chi fall RCTs in older persons with cognitive impairment, and the remaining reviews did not provide a summary effect estimate. Of note, reviews in hospitals and nursing home settings (Falls-institutions) did not report positive findings $[9,17]$.

One systematic review on the effects of Tai Chi on cognitive performance in older adults identified 11 relevant RCTs [100]. This review found positive effects of Tai Chi on executive function in cognitively healthy adults compared to no intervention (SMD 0.90; $p=0.04 ; 4$ RCTs) and exercise (SMD 0.51; $p=0.003 ; 2$ RCTs), on global cognitive function in cognitively impaired adults compared with no intervention (SMD 0.35; $p=0.004 ; 4$ RCTs) or other active interventions (SMD 0.30; $p=0.002$; 4 RCTs). However, it cautioned that larger and methodologically sound trials with longer follow-up periods are needed before definitive conclusions can be drawn.

There are also a number of areas suggesting promising results but for which the volume of research is smaller and fewer than ten relevant RCTs were available to inform the reviews. Eight systematic reviews have addressed osteoarthritis [5, 12, 21, 30,44, 63, 88, 90, 103], and the two largest reviews included nine RCTs each. One of them reported pooled results and showed positive effects of Tai Chi compared to different control groups on pain (SMD $-0.79 ; 95 \%$ CI $-1.19,-0.39$; 6 RCTs), physical function (SMD -0.86; $95 \%$ CI $-1.20,-0.52 ; 6$ RCTs), and joint stiffness (SMD -0.53; $95 \%$ CI $-0.99,-0.08$; 6 RCTs) but cautioned that due to the small number of RCTs with a low risk of bias, the evidence that Tai Chi is effective in patients with osteoarthritis is limited [5]. An independent review reported significant positive short-term effects for pain intensity (SMD $-0.72 ; 95 \% \mathrm{CI}-1.00,-0.44 ; 5 \mathrm{RCTs}$ ), function (SMD $-0.72 ; 95 \%$ CI $-1.01,-0.44 ; 5 \mathrm{RCTs}$ ), stiffness (SMD $-0.59 ; 95 \%$ CI $-0.99,-0.19 ; 5$ RCTs), and physical quality of life (SMD 0.88; $95 \%$ CI 0.42, 1.34; 2 RCTs) but not for mental quality of life, or long-term effects for pain, physical function, and stiffness, compared to waitlist or attention control. The authors highlighted that all positive results represent short-term effects and high-quality RCTs are needed to confirm the results [63]. 
A 2013 meta-analysis reported statistically significant and clinically important effects for pain (SMD -0.45; $95 \%$ CI $-0.70,-0.20$; 7 RCTs) across studies comparing Tai Chi to waiting list, Bingo, attention control programs, routine treatment, self-help programs, or wellness education and stretching, and concluded that 12-week Tai Chi programs should be included in rehabilitation programs but highlighted that the pain - relieving effect is not sustained and that additional studies are needed to investigate the long-term effects of Tai Chi in patients with knee osteoarthritis [103]. The remaining reviews did not identify eligible Tai Chi RCTs for their particular review question or did not report treatment effects across studies.

Positive outcomes were also reported in two reviews on chronic obstructive pulmonary disease (COPD) [42, 104] and the largest included eight RCTs. The largest review reported statistically significant pooled effects of Tai Chi for the 6-min walk test (WMD $34.22 \mathrm{~m}$; $95 \%$ CI 21.25, 47.20; 3 RCTs), dyspnea (WMD -0.86; $95 \%$ CI $-1.44,-0.28,3$ RCTs), forced expiratory volume in $1 \mathrm{~s}$ (WMD 0.07; $95 \%$ CI 0.02, 0.13, 4 RCTs), forced vital capacity (WMD 0.12; 95 \% CI 0.00, 0.23, 3 RCTs), and two quality of life measures (WMD 0.95; $95 \% \mathrm{CI}$ 0.22, 1.67; 2 RCTs; WMD -4.08; 95 \% CI -7.52, -0.64; 3 RCTs), comparator not specified [104]. The second review combined Tai Chi and qigong interventions and did not provide treatment estimates across Tai Chi studies. Four systematic reviews have focused on the outcome pain $[49,81,107,112]$ and the largest review included seven RCTs (including six arthritis RCTs). The largest review found a positive effect of Tai Chi on selfreported pain (WMD 10.1 points on a $0-100$ scale; $95 \%$ CI 6.3, 13.9; 6 RCTs; comparators not specified) and selfreported disability (WMD -9.6; $95 \%$ CI $-14,-5.2 ; 4$ RCTs) but not for physical performance, and data for quality of life were not pooled across studies [49]. Pooled treatment estimates of Tai Chi across studies were not reported in two other reviews, and one review found no eligible Tai Chi RCT. Five systematic reviews focused on balance confidence/fear of falling $[15,18,33,83,91]$ and the largest included six RCTs. One reported a positive effect for Tai Chi compared to usual care, exercise, or education (SMD 0.47; $95 \%$ CI 0.30, 0.63; 4 RCTs) [83]. The other reviews did not report a treatment effect estimate across studies. Five systematic reviews have specifically addressed the effects of Tai Chi on depression; [26, 34, 38, 93, 102] the largest review included four RCTs. It reported statistically significantly reduced depression symptoms (SMD -0.27; $95 \%$ CI -0.52; -0.02; 4 RCTs) compared to waitlist in older adults but highlighted that further research is recommended with larger samples sizes, more clarity on trial design and the intervention, longer-term follow-up, and concomitant economic evaluations [38]. The other depression-specific reviews included only one or two Tai Chi studies or did not distinguish effects attributable to Tai Chi. However, the review of psychological well-being included nine RCTs reporting on depression, and it also reported a positive effect (Hedges' $g$ 0.48; $95 \%$ CI 0.17, 0.78) [95]. A review on lower limb muscle strength in the elderly included two RCTs; both reported positive effects but did not report on the same outcome $[11,114]$.

\section{Evidence of no effect and unclear or conflicting evidence} The map includes a small number of systematic reviews that provide evidence of the potential lack of effectiveness of Tai Chi for clinical indications across more than one included study, e.g., fall prevention in hospitals and nursing homes (see the left hand side of the map). For these topics, systematic review authors concluded across identified studies that Tai Chi did not improve outcomes of interest; however, the number of existing studies in the identified topic areas was small in all of the identified topic areas.

In addition, unclear or conflicting evidence was found for a large number of topical areas as shown in the large middle section of the evidence map; in some cases, despite a number of existing systematic reviews that have attempted to synthesize the evidence in the research area.

\section{Lack of research}

The evidence map also shows clinical topics that have been reviewed, but for which no Tai Chi studies could be found $(y$-axis $=0)$. Systematic reviews on menopause, dementia, metabolic syndrome, post-traumatic stress disorder (PTSD), urinary incontinence, multiple sclerosis, and anxiety during pregnancy systematically searched for Tai Chi studies. However, no RCTs, i.e., research studies supporting a high level of evidence, were identified in these systematic reviews.

\section{Other evidence base variables}

Of the 107 included reviews, $42 \%$ reported on the presence or absence of adverse events (not shown in Fig. 2). The large majority of these reviews noted that Tai Chi had little or no adverse effects on study participants. However, doing any exercise may put participants at greater risk and one review concluded that Tai Chi practiced by older adults may only be effective in a more robust older population and may not benefit frail participants [48]. None of the included reviews was exclusively based on Tai Chi interventions that deviated from traditional formats.

\section{Discussion}

This evidence map for Tai Chi is based on 107 published systematic reviews and provides a broad overview of the 
available evidence of Tai Chi and its effect on patient outcomes. It shows the research concentration and the volume of available research and highlights areas where published meta-analyses have reported positive results.

Tai Chi has been evaluated for a wide range of clinical applications. Some identified systematic reviews included a large number of RCTs, but they addressed very broad topics such as health effects, psychological well-being, or interventions targeting older adults. On the other hand, evidence on the role of Tai Chi for a number of specific conditions is very limited due to the small number of published studies. Two thirds of identified systematic reviews included in this map were published very recently, i.e., in the last five years.

Although evidence maps can only provide a broad overview of research areas, it is noteworthy that across clinical topic areas, reviews concluded that more rigorous research on the clinical effectiveness of Tai Chi is needed. Furthermore, the effectiveness of Tai Chi may depend on several different factors including setting or patient characteristics-as indicated by differential effects of fall prevention in community versus hospitals or nursing homes. The optimal range of the Tai Chi intervention duration (short term versus long term) has not been determined, and a number of authors have indicated that more research on long-term effects is needed [63].

Our review of reviews also found that adverse events of Tai Chi have not been investigated systematically as noted in a recent review [115]. Given that the quality of the reporting of adverse events may depend on the standards in individual clinical fields, analyses across large, multi-indication reviews are particularly useful; a recent review concluded that much can be learned by comparing the effects of a given treatment across many related indications [114].

The evidence map-a visual overview of a systematic review of systematic reviews-is a new and unique review product that shows graphically, at a glance, the volume and focus of a research area through bubble color, size, and location. Based on a delineated systematic process (e.g., having specified search and inclusion criteria), evidence maps can be used to identify knowledge gaps and future research needs and to provide easily digestible and usable information from a large body of literature. Because evidence mapping is a relatively new and innovative evidence synthesis method, there are no established reporting guidelines; however, some principles have been articulated, including the use of an expert panel to ensure relevance and usefulness of the evidence, such as were used in this review [4].

The evidence map has several limitations. First, evidence maps cannot provide definitive answers about the effectiveness of an intervention. We used published reviews to provide an overview over the research on
Tai Chi and did not undertake independent systematic reviews to calculate effect sizes in a meta-analysis, provide risk of bias assessments, or establish quality of evidence evaluations ourselves. Furthermore, the unit of analysis was systematic reviews, and individual primary research studies will have contributed to more than one included systematic review, in particular as reviews focused on different clinical indications, outcomes, or populations. In addition, the grouping of systematic reviews was review-content driven. The map did not follow a predefined structure and was unable to avoid overlap between included studies across reviews; the map was based on published reviews and used the topic structure of the reviews in order to explore the evidence base. The evidence map used review-level data, not primary research study data, and relied on the review authors' clinical topic interest and skill in conducting systematic reviews. Furthermore, individual review conclusions may be limited by the quality of primary studies and susceptible to publication and outcome reporting bias.

Included Tai Chi interventions varied greatly by Tai Chi style, intervention duration, and intervention intensity; and studies varied in their choice of comparator to estimate the effectiveness of Tai Chi. A broad overview cannot answer more refined questions such as the effect of different styles of Tai Chi, the effect of the practitioner's training, and skill level or the role of patients' Tai Chi practice efforts. More specific results need targeted systematic reviews (addressing selected clinical indications and outcomes) and effect modifiers should be analyzed in meta-regressions designed to identify sources of heterogeneity across studies.

Future research should consider the body of evidence assembled in this map and systematically explore the effects of Tai Chi on clinically relevant outcomes across identified reviews. This broad overview has explored the research focus, as described in existing systematic reviews, and the map has identified several promising areas. Establishing more information on the effects of Tai Chi across and within clinical indications and patient populations, through meta-analyses across primary research studies, will further advance our evidence-based knowledge of Tai Chi. In addition, the large number of topic areas that were classified as unclear evidence warrants further research. Some topics addressed in reviews were very broad ("health," "psychological wellbeing," "older adults," or "cancer") and would benefit from targeted syntheses for specific outcomes. In other areas, there is a clear need for additional primary studies. The lack of positive or negative effect estimates is primarily a function of the absence of studies (Tai Chi effects on menopause, multiple sclerosis, metabolic syndrome, PTSD, dementia, urinary incontinence, asthma, 
and anxiety in pregnancy) at the time of the review. Finally, reviews for some of the topic areas included in the unclear evidence category have identified an emerging body of research, but summary effects estimating the treatment effect of Tai Chi are missing and should be addressed in future meta-analyses.

\section{Conclusions}

Tai Chi has been applied in diverse clinical areas, and for a number of these, systematic reviews have indicated promising results. The evidence map provides a visual overview of Tai Chi research volume and content. Despite the outlined limitations, evidence maps provide valuable information on the landscape-the size, scope, and breadth-of a given domain of research. The visualization facilitates an easy and engaging overview and suggests evidence maps as a tool useful for a large array of stakeholders and for informing policy and clinical decision makers.

\section{Additional files}

Additional file 1: PRISMA checklist.

Additional file 2: Search strategy.

\section{Acknowledgements}

We would like to thank the technical expert panel advising on the project: Stephen Ezeji-Okoye, Laura Krejci, Peter Asco, Ansgar Furst, Laura Redwine, Greg Patterson, and Elmer Ligh. We also thank Andrew Siroka for assistance with designing the bubble plot, Aneesa Motala for editorial assistance, Ning Fu for assistance with the data extraction, and Jeremy Miles for assistance with the data synthesis. Any errors of fact or interpretation in this manuscript remain the responsibility of the authors.

\section{Funding}

The study is based on a systematic review conducted by the Evidence-based Synthesis Program (ESP) funded by the Department of Veterans Affairs (VA). The funding source commissioned the study as an evidence map but other than that had no role in the conduct of the study; collection, management, analysis, and interpretation of the data; preparation, review, or approval of the manuscript; and decision to submit the manuscript for publication. The findings and conclusions in this publication are those of the authors who are responsible for its contents; the findings and conclusions do not necessarily represent the views of the VA or the ESP program.

\section{Availability of data and materials}

All included systematic reviews are in the public domain; see the "Reference" section for full citation details. Study flow was tracked in citation management software and data were extracted in an online systematic review program and in Excel; all files can be obtained from the authors.

\section{Authors' contributions}

MS and SH drafted the manuscript. SH, PS, and IML designed the study. RS designed and executed the search strategy. JB, IML, MS, SH, and ST were involved in data acquisition and analysis. All authors were involved in the interpretation of the data and contributed to the final manuscript All authors read and approved the final manuscript.

\section{Competing interests}

PS is a Co-Editor in Chief, and SH is an Associate Editor of Systematic Reviews.
Consent for publication

Not applicable

Ethics approval and consent to participate

Not applicable.

\section{Author details}

${ }^{1}$ VA Greater Los Angeles Healthcare System, Los Angeles, CA, USA. ${ }^{2}$ VA Evidence-based Synthesis Program (ESP) Center, Los Angeles, CA, USA. ${ }^{3}$ University of California, Los Angeles, CA, USA. ${ }^{4}$ Evidence-based Practice Center (EPC), RAND Corporation, Santa Monica, CA, USA.

Received: 23 January 2016 Accepted: 14 July 2016

Published online: 27 July 2016

\section{References}

1. NIH National Center for Complementary and Integrative Health (formerly the National Center for Complementary and Alternative medicine). Tai Chi: an introduction. 2010

2. Lee MS, Ernst E. Systematic reviews of t'ai chi: an overview. Br I Sports Med. 2012;46:713-8.

3. Hempel S, Taylor SL, Solloway MR, Miake-Lye IM, Beroes JM, et al. Evidence map of Tai Chi. Washington (DC): Department of Veterans Affairs (US); 2014.

4. Hetrick SE, Parker AG, Callahan P, Purcell R. Evidence mapping: illustrating an emerging methodology to improve evidence-based practice in youth mental health. J Eval Clin Pract. 2010;16:1025-30.

5. Kang JW, Lee MS, Posadzki P, Ernst E. T'ai chi for the treatment of osteoarthritis: a systematic review and meta-analysis. BMJ Open. 2011;1:e000035.

6. Lee MS, Pittler MH, Ernst E. Tai chi for osteoarthritis: a systematic review. Clin Rheumatol. 2008:27:211-8.

7. Ospina MB, Bond K, Karkhaneh M, Tjosvold L, Vandermeer B, et al. Meditation practices for health: state of the research. Evidence report/ technology assessment. 2007. p. 1-263.

8. Ospina MB, Bond K, Karkhaneh M, Buscemi N, Dryden DM, et al. Clinical trials of meditation practices in health care: characteristics and quality. J Altern Complement Med. 2008;14:1199-213.

9. Cameron ID, Murray GR, Gillespie LD, Robertson MC, Hill KD, et al. Interventions for preventing falls in older people in nursing care facilities and hospitals. Cochrane Database Syst Rev. 2012;1:CD005465.

10. Leung DP, Chan CK, Tsang HW, Tsang WW, Jones AY. Tai chi as an intervention to improve balance and reduce falls in older adults: a systematic and metaanalytical review. Altern Ther Health Med. 2011;17:40-8.

11. Liu B, Liu ZH, Zhu HE, Mo JC, Cheng DH. Effects of tai chi on lower-limb myodynamia in the elderly people: a meta-analysis. J Tradit Chin Med. 2011;31:141-6.

12. Uthman OA, van der Windt DA, Jordan UL, Dziedzic KS, Healey EL, et al. Exercise for lower limb osteoarthritis: systematic review incorporating trial sequential analysis and network meta-analysis. BMJ. 2013;347:f5555.

13. Woods NF, Mitchell ES, Schnall JG, Cray L, Ismail R, et al. Effects of mindbody therapies on symptom clusters during the menopausal transition. Climacteric. 2014;17:10-22

14. Bo K, Herbert RD. There is not yet strong evidence that exercise regimens other than pelvic floor muscle training can reduce stress urinary incontinence in women: a systematic review. J Physiother. 2013: 59:159-68.

15. Bula CJ, Monod S, Hoskovec C, Rochat S. Interventions aiming at balance confidence improvement in older adults: an updated review. Gerontology. 2011;57:276-86

16. Chen KM, Snyder M. A research-based use of Tai Chi/movement therapy as a nursing intervention. J Holist Nurs. 1999;17:267-79.

17. Church J, Goodall S, Norman R, Haas M. An economic evaluation of community and residential aged care falls prevention strategies in NSW. N S W Public Health Bull. 2011;22:60-8.

18. Lach HW, Parsons JL. Impact of fear of falling in long term care: an integrative review. J Am Med Dir Assoc. 2013;14:573-7.

19. Lee MS, Pittler MH, Taylor-Piliae RE, Ernst E. Tai chi for cardiovascular disease and its risk factors: a systematic review. J Hypertens. 2007;25:1974-5.

20. Michael YL, Lin JS, Whitlock EP, Gold R, Fu R, et al. Interventions to prevent falls in older adults: an updated systematic review. Evidence report no. 80. AHRQ publication no. 11-05150-EF-1. Rockville: Agency for Healthcare Research and Quality; 2010 
21. Shengelia R, Parker SJ, Ballin M, George T, Reid MC. Complementary therapies for osteoarthritis: are they effective? Pain Manag Nurs. 2013;14:e274-288.

22. Toh SFM. A systematic review on the effectiveness of Tai Chi exercise in individuals with Parkinson's disease from 2003 to 2013. Hong Kong J Occup Ther. 2014;23(2):69-81.

23. van der Heijden MMP, van Dooren FEP, Pop VJM, Pouwer F. Effects of exercise training on quality of life, symptoms of depression, symptoms of anxiety and emotional well-being in type 2 diabetes mellitus: a systematic review. Diabetologia. 2013;56:1210-25.

24. Wang F, Lee EK, Wu T, Benson H, Fricchione G, et al. The effects of Tai Chi on depression, anxiety, and psychological well-being: a systematic review and meta-analysis. Int J Behav Med. 2013;21(4):605-617.

25. Yan JH, Gu WJ, Pan L. Lack of evidence on Tai Chi-related effects in patients with type 2 diabetes mellitus: a meta-analysis. Exp Clin Endocrinol Diabetes. 2013;121:266-71.

26. Yohannes AM, Caton S. Management of depression in older people with osteoarthritis: a systematic review. Aging Ment Health. 2010;14(6):637-51.

27. Zeng $Y$, Luo $T$, Xie H, Huang M, Cheng ASK. Health benefits of qigong or tai chi for cancer patients: a systematic review and meta-analyses. Complement Ther Med. 2014;22(1):173-86.

28. Zhang F, Kong LL, Zhang YY, Li SC. Evaluation of impact on health-related quality of life and cost effectiveness of Traditional Chinese Medicine: a systematic review of randomized clinical trials. J Altern Complement Med. 2012;18:1108-20.

29. Anderson JG, Taylor AG. The metabolic syndrome and mind-body therapies: a systematic review. J Nutr Metab. 2011;2011:276419.

30. Escalante Y, Garcia-Hermoso A, Saavedra JM. Effects of exercise on functional aerobic capacity in lower limb osteoarthritis: a systematic review. J Sci Med Sport. 2011;14:190-8.

31. Marc I, Toureche N, Ernst E, Hodnett ED, Blanchet C, et al. Mind-body interventions during pregnancy for preventing or treating women's anxiety. Cochrane Database Syst Rev. 2011;7:CD007559.

32. Wang WC, Zhang AL, Rasmussen B, Lin LW, Dunning T, et al. The effect of Tai Chi on psychosocial well-being: a systematic review of randomized controlled trials. J Acupunct Meridian Stud. 2009;2:171-81.

33. Zijlstra GA, van Haastregt JC, van Rossum E, van Eijk JT, Yardley L, et al. Interventions to reduce fear of falling in community-living older people: a systematic review. J Am Geriatr Soc. 2007;55:603-15.

34. Bridle C, Spanjers K, Patel S, Atherton NM, Lamb SE. Effect of exercise on depression severity in older people: systematic review and meta-analysis of randomised controlled trials. Br J Psychiatry. 2012;201(3):180-5.

35. Brown JC, Huedo-Medina TB, Pescatello LS, Pescatello SM, Ferrer RA, Johnson BT. Efficacy of exercise interventions in modulating cancer-related fatigue among adult cancer survivors: a meta-analysis. Cancer Epidemiol Biomark Prev. 2011;20(1):123-33.

36. Bu B, Haijun $H$, Yong $L$, Chaohui $Z$, Xiaoyuan $Y$, et al. Effects of martial arts on health status: a systematic review. J Evid Based Med. 2010;3:205-19.

37. Chan WW, Bartlett DJ. Effectiveness of Tai Chi as a therapeutic exercise in improving balance and postural control. Phys Occup Ther Geriatr. 2000;17:1-22

38. Chi I, Jordan-Marsh M, Guo M, Xie B, Bai Z. Tai chi and reduction of depressive symptoms for older adults: a meta-analysis of randomized trials. Geriatr Gerontol Int. 2013;13:3-12

39. Conrad Wooton A. An integrative review of Tai Chi research: an alternative form of physical activity to improve balance and prevent falls in older adults (Provisional abstract). Orthop Nurs. 2010;29:108-118.

40. Dalusung-Angosta A. The impact of Tai Chi exercise on coronary heart disease: a systematic review. J Am Acad Nurse Pract. 2011;23:376-81.

41. Ding M. Tai Chi for stroke rehabilitation: a focused review. Am J Phys Med Rehabil. 2012;91:1091-6.

42. Ding M, Zhang W, Li K, Chen X. Effectiveness of T'ai Chi and Qigong on chronic obstructive pulmonary disease: a systematic review and metaanalysis. 2013. J Altern Complement Med.

43. El-Khoury F, Cassou B, Charles MA, Dargent-Molina P. The effect of fall prevention exercise programmes on fall induced injuries in community dwelling older adults: systematic review and meta-analysis of randomised controlled trials. BMJ. 2013;347:f6234.

44. Escalante Y, Saavedra JM, Garcia-Hermoso A, Silva AJ, Barbosa TM. Physical exercise and reduction of pain in adults with lower limb osteoarthritis: a systematic review. J Back Musculoskelet Rehabil. 2010;23:175-86.
45. Fairhall N, Sherrington C, Clemson L, Cameron ID. Do exercise interventions designed to prevent falls affect participation in life roles? A systematic review and meta-analysis. Age Ageing. 2011;40(6):666-74.

46. Forbes D, Thiessen EJ, Blake CM, Forbes SC, Forbes S. Exercise programs for people with dementia. Cochrane Database Syst Rev. 2013;12:CD006489.

47. Gillespie LD, Robertson MC, Gillespie WJ, Sherrington C, Gates S, et al. Interventions for preventing falls in older people living in the community. Cochrane Database Syst Rev. 2012;9:CD007146.

48. Gregory H, Watson MC. The effectiveness of Tai Chi as a fall prevention intervention for older adults: a systematic review (Structured abstract). Int J Health Promot Educ. 2009:47:94-100.

49. Hall A, Maher C, Latimer J, Ferreira M. The effectiveness of Tai Chi for chronic musculoskeletal pain conditions: a systematic review and metaanalysis. Arthritis Rheum. 2009;61:717-24.

50. Han A, Robinson V, Judd M, Taixiang W, Wells G, et al. Tai chi for treating rheumatoid arthritis. Cochrane Database Syst Rev. 2004;3:CD004849.

51. Harling A, Simpson JP. A systematic review to determine the effectiveness of Tai Chi in reducing falls and fear of falling in older adults (Structured abstract). Phys Ther Rev. 2008;13:237-248.

52. Ho RT, Wang CW, Ng SM, Ho AH, Ziea ET, et al. The effect of t'ai chi exercise on immunity and infections: a systematic review of controlled trials. J Altern Complement Med. 2013;19:389-96.

53. Howe TE, Rochester $L$, Neil F, Skelton DA, Ballinger C. Exercise for improving balance in older people. Cochrane Database Syst Rev. 2011;11:CD004963.

54. Innes KE, Selfe TK, Vishnu A. Mind-body therapies for menopausal symptoms: a systematic review. Maturitas. 2010;66:135-49.

55. Jahnke R, Larkey L, Rogers C, Etnier J, Lin F. A comprehensive review of health benefits of qigong and tai chi. Am J Health Promot. 2010;24:e1-e25.

56. Jimenez PJ, Melendez A, Albers U. Psychological effects of Tai Chi Chuan. Arch Gerontol Geriatr. 2012;55:460-7.

57. Jimenez-Martin PJ, Melendez-Ortega A, Albers U, Schofield D. A review of Tai Chi Chuan and parameters related to balance. Eur J Integr Med. 2013:5:469-75.

58. Kim SH, Schneider SM, Kravitz L, Mermier C, Burge MR. Mind-body practices for posttraumatic stress disorder. J Investig Med. 2013;61:827-34.

59. Klein PJ, Adams WD. Comprehensive therapeutic benefits of Taiji: a critical review. Am J Phys Med Rehabil. 2004;83:735-45.

60. Klein PT, Adams W. Cardiopulmonary physiotherapeutic applications of taiji (Structured abstract). Cardiopulm Phys Ther. 2004;15:5-11.

61. Komagata S, Newton R. The effectiveness of Tai Chi on improving balance in older adults: an evidence-based review (Provisional abstract). J Geriatr Phys Ther. 2003;26:9-16.

62. Langhorst J, Klose P, Dobos GJ, Bernardy K, Hauser W. Efficacy and safety of meditative movement therapies in fibromyalgia syndrome: a systematic review and meta-analysis of randomized controlled trials. Rheumatol Int 2013;33:193-207.

63. Lauche R, Langhorst J, Dobos G, Cramer H. A systematic review and metaanalysis of Tai Chi for osteoarthritis of the knee. Complement Ther Med. 2013;21:396-406.

64. Lee MS, Choi TY, Ernst E. Tai chi for breast cancer patients: a systematic review. Breast Cancer Res Treat. 2010;120:309-16.

65. Lee MS, Choi TY, Lim HJ, Ernst E. Tai chi for management of type 2 diabetes mellitus: a systematic review. Chin J Integr Med. 2011;17:789-93.

66. Lee MS, Lam P, Ernst E. Effectiveness of tai chi for Parkinson's disease: a critical review. Parkinsonism Relat Disord. 2008;14:589-94.

67. Lee MS, Lee EN, Ernst E. Is tai chi beneficial for improving aerobic capacity? A systematic review. Br J Sports Med. 2009:43:569-73.

68. Lee MS, Lee EN, Kim Jl, Ernst E. Tai chi for lowering resting blood pressure in the elderly: a systematic review. J Eval Clin Pract. 2010;16:818-24.

69. Lee MS, Pittler MH, Ernst E. Tai chi for rheumatoid arthritis: systematic review. Rheumatology (Oxford). 2007:46:1648-51.

70. Lee MS, Pittler MH, Ernst E. Is Tai Chi an effective adjunct in cancer care? A systematic review of controlled clinical trials. Support Care Cancer. 2007;15:597-601.

71. Lee MS, Pittler MH, Shin BC, Ernst E. Tai chi for osteoporosis: a systematic review. Osteoporos Int. 2008:19:139-46.

72. Liu H, Frank A. Tai chi as a balance improvement exercise for older adults: a systematic review. J Geriatr Phys Ther. 2010;33:103-9.

73. Logghe $I H$, Verhagen AP, Rademaker AC, Bierma-Zeinstra SM, van Rossum E, et al. The effects of Tai Chi on fall prevention, fear of falling and balance in older people: a meta-analysis. Prev Med. 2010;51:222-7. 
74. Lorenc AB, Wang Y, Madge SL, Hu X, Mian AM, et al. Meditative movement for respiratory function: a systematic review. Respir Care. 2013;59(3):427-440.

75. Low S, Ang LW, Goh KS, Chew SK. A systematic review of the effectiveness of Tai Chi on fall reduction among the elderly. Arch Gerontol Geriatr. 2009; 48:325-31.

76. Macfarlane GJ, Paudyal P, Doherty M, Ernst E, Lewith G, et al. A systematic review of evidence for the effectiveness of practitioner-based complementary and alternative therapies in the management of rheumatic diseases: rheumatoid arthritis. Rheumatology (Oxford). 2012;51:1707-13.

77. Maciaszek J, Osinski W. The effects of Tai Chi on body balance in elderly people - a review of studies from the early 21st century. Am J Chin Med. 2010;38:219-29.

78. Mansky P, Sannes T, Wallerstedt D, Ge A, Ryan M, et al. Tai chi chuan: mindbody practice or exercise intervention? Studying the benefit for cancer survivors (Structured abstract). Integr Cancer Ther. 2006;5:192-201.

79. Mishra SI, Scherer RW, Geigle PM, Berlanstein DR, Topaloglu O, et al. Exercise interventions on health-related quality of life for cancer survivors. Cochrane Database Syst Rev. 2012;8:CD007566.

80. Mist SD, Firestone KA, Jones KD. Complementary and alternative exercise for fibromyalgia: a meta-analysis. J Pain Res. 2013;6:247-60.

81. Morone NE, Greco CM. Mind-body interventions for chronic pain in older adults: a structured review. Pain Med. 2007:8:359-75.

82. Ng SM, Wang CW, Ho RT, Ziea TC, He J, et al. Tai chi exercise for patients with heart disease: a systematic review of controlled clinical trials. Altern Ther Health Med. 2012;18:16-22.

83. Rand D, Miller WC, Yiu J, Eng JJ. Interventions for addressing low balance confidence in older adults: a systematic review and meta-analysis. Age Ageing. 2011;40:297-306

84. Rogers C, Keller C, Larkey LK. Perceived benefits of meditative movement in older adults. Geriatr Nurs. 2010;31:37-51.

85. Rogers CE, Larkey LK, Keller C. A review of clinical trials of tai chi and qigong in older adults. West J Nurs Res. 2009;31:245-79.

86. Sarris J, Byrne GJ. A systematic review of insomnia and complementary medicine. Sleep Med Rev. 2011;15:99-106.

87. Schleicher MM, Wedam L, Wu G. Review of Tai Chi as an effective exercise on falls prevention in elderly. Res Sports Med. 2012;20:37-58.

88. Selfe TK, Innes KE. Mind-body therapies and osteoarthritis of the knee. Cur Rheumatol Rev. 2009:5:204-11.

89. Sharma M, Haider T. Tai chi as an alternative and complementary therapy for patients with asthma and chronic obstructive pulmonary disease: a systematic review. J Evid Based Complement Altern Med. 2013;18:209-15.

90. Simic M, Hinman RS, Wrigley TV, Bennell KL, Hunt MA. Gait modification strategies for altering medial knee joint load: a systematic review. Arthritis Care Res (Hoboken). 2011:63:405-26.

91. Sjosten N, Vaapio S, Kivela SL. The effects of fall prevention trials on depressive symptoms and fear of falling among the aged: a systematic review. Aging Ment Health. 2008;12:30-46.

92. Taylor-Piliae RE. The effectiveness of Tai Chi exercise in improving aerobic capacity: an updated meta-analysis. Med Sport Sci. 2008;52:40-53.

93. Tsang HW, Chan EP, Cheung WM. Effects of mindful and non-mindful exercises on people with depression: a systematic review. Br J Clin Psychol. 2008;47:303-22.

94. Verhagen AP, Immink M, van der Meulen A, Bierma-Zeinstra SM. The efficacy of Tai Chi Chuan in older adults: a systematic review. Fam Pract. 2004:21:107-13

95. Wang C, Bannuru R, Ramel J, Kupelnick B, Scott T, et al. Tai Chi on psychological well-being: systematic review and meta-analysis. BMC Complement Altern Med. 2010;10:23.

96. Wang C, Collet JP, Lau J. The effect of Tai Chi on health outcomes in patients with chronic conditions: a systematic review. Arch Intern Med. 2004;164:493-501

97. Wang J, Feng B, Yang X, Liu W, Teng F, et al. Tai chi for essential hypertension. Evid Based Complement Alternat Med. 2013:2013:215254.

98. Wayne PM, Kiel DP, Krebs DE, Davis RB, Savetsky-German J, et al. The effects of Tai Chi on bone mineral density in postmenopausal women: a systematic review. Arch Phys Med Rehabil. 2007:88:673-80.

99. Wayne PM, Krebs DE, Wolf SL, Gill-Body KM, Scarborough DM, et al. Can Tai Chi improve vestibulopathic postural control? Arch Phys Med Rehabil. 2004;85:142-52
100. Wayne PM, Walsh JN, Taylor-Piliae RE, Wells RE, Papp KV, et al. Effect of Tai Chi on cognitive performance in older adults: systematic review and metaanalysis. J Am Geriatr Soc. 2014;62(1):25-39.

101. Winter $H$, Watt $K$, Peel NM. Falls prevention interventions for communitydwelling older persons with cognitive impairment: a systematic review. Int Psychogeriatr. 2013;25(2):215-27.

102. Woltz PC, Chapa DW, Friedmann E, Son H, Akintade B, et al. Effects of interventions on depression in heart failure: a systematic review. Heart Lung. 2012:41:469-83.

103. Yan JH, Gu WJ, Sun J, Zhang WX, Li BW, et al. Efficacy of Tai Chi on pain, stiffness and function in patients with osteoarthritis: a meta-analysis. PLoS One. 2013;8:e61672

104. Yan JH, Guo YZ, Yao HM, Pan L. Effects of Tai Chi in patients with chronic obstructive pulmonary disease: preliminary evidence. PLoS One. 2013;8:e61806.

105. Yeh GY, Wang C, Wayne PM, Phillips R. Tai chi exercise for patients with cardiovascular conditions and risk factors: a systematic review. J Cardiopulm Rehabil Prev. 2009;29:152-60.

106. Yeh GY, Wang C, Wayne PM, Phillips RS. The effect of tai chi exercise on blood pressure: a systematic review. Prev Cardiol. 2008;11:82-9.

107. Reid MC, Papaleontiou M, Ong A, Breckman R, Wethington E, et al. Self-management strategies to reduce pain and improve function among older adults in community settings: a review of the evidence. Pain Med. 2008;9:409-24

108. Pan L, Yan J, Guo Y, Yan J. Effects of Tai Chi training on exercise capacity and quality of life in patients with chronic heart failure: a meta-analysis. Eur J Heart Fail. 2013;15:316-23.

109. Wu G. Progress in geriatrics Evaluation of the effectiveness of Tai Chi for improving balance and preventing falls in the older population-a review. J Am Geriatr Soc. 2002;50:746-54.

110. Binns EE, Taylor D. Does Tai Chi improve strength and balance in people with multiple sclerosis - the current literature. N Z J Physiother. 2008;36:83.

111. Dechamps A, Lafont L, Bourdel-Marchasson I. Effects of Tai Chi exercises on self-efficacy and psychological health. Eur Rev Aging Phys Act. 2007;4:25-32.

112. Wang XQ, Zheng JJ, Yu ZW, Bi X, Lou SJ, et al. A meta-analysis of core stability exercise versus general exercise for chronic low back pain. PLoS One. 2012;7:e52082

113. Windle G, Hughes D, Linck P, Russell I, Woods B. Is exercise effective in promoting mental well-being in older age? A systematic review. Aging Ment Health. 2010;14:652-69.

114. Chen YF, Hemming K, Chilton PJ, Gupta KK, Altman DG, et al. Scientific hypotheses can be tested by comparing the effects of one treatment over many diseases in a systematic review. J Clin Epidemiol. 2014;67:1309-19.

115. Wayne PM, Berkowitz DL, Litrownik DE, Buring JE, Yeh GY. What do we really know about the safety of Tai Chi?: a systematic review of adverse event reports in randomized trials. Arch Phys Med Rehabil. 2014;95:2470-83.

\section{Submit your next manuscript to BioMed Central and we will help you at every step:}

- We accept pre-submission inquiries

- Our selector tool helps you to find the most relevant journal

- We provide round the clock customer support

- Convenient online submission

- Thorough peer review

- Inclusion in PubMed and all major indexing services

- Maximum visibility for your research

Submit your manuscript at www.biomedcentral.com/submit 